\title{
COMPORTAMIENTO AGRONÓMICO DE LÍNEAS PROMISORIAS DE FRÍJOL CAUPÍ Vigna unguiculata L. Walp EN EL VALLE DEL SINÚ
}

\section{AGRONOMIC BEHAVIOR OF PROMISING LINES OF COWPEA BEANS Vigna unguiculata L. Walp IN SINU VALLEY}

\author{
Hermes Araméndiz-Tatis ${ }^{1}$, Miguel Espitia-Camachoํ, Carlos M. Sierra² \\ Recibido para publicación: Agosto 19 de 2011 - Aceptado para publicación: Diciembre 22 de 2011
}

\begin{abstract}
RESUMEN
Se evaluó el comportamiento agronómico de 12 líneas promisorias de fríjol caupí y la población original con el fin de seleccionar genotipos superiores en atributos agronómicos teniendo en cuenta las condiciones agroecológicas del Valle del Sinú, departamento de Córdoba, Colombia. El diseño de bloques completos al azar con 13 tratamientos y cuatro repeticiones fue aplicado. Las variables de respuesta fueron el rendimiento de grano y sus componentes. La siembra se realizó en el área experimental de la Universidad de Córdoba localizada en Montería - Colombia, en el año 2009. El análisis de varianza detectó diferencias significativas $(\mathrm{P} \leq 0,05)$ para el número de vainas por planta, vainas por metro lineal, número de semillas por vaina y rendimiento de grano, mientras que para el peso de semilla se presentaron diferencias altamente significativa $(\mathrm{P} \leq 0,01)$. Caso contrario ocurrió para la precocidad donde el análisis acusó ausencia de significancia. La variabilidad genética presente en las líneas endogámicas posibilita seleccionar cultivares con buenos atributos agronómicos y excelentes rendimientos de grano que mejoren los niveles de producción de los agricultores en el Valle del Sinú.
\end{abstract}

Palabras Clave: Caupí, rendimiento de grano, población parental.

\begin{abstract}
The agronomic performance of 12 cowpea genotypes and the original population was evaluated in order to select genotypes with higher agronomic attributes, taking into account the agro-ecological conditions of the Sinú river valley, departament of Cordoba, Colombia. Randomized complete block design with 13 treatments and four replications was applied. Response variables were grain yield and its components. The populations

Universidad de Córdoba. Departamento de Ingeniería Agronómica y Desarrollo Rural. Carrera 6 №76-103, Montería Colombia. Email: haramendiz@hotmail.com; mespitia@hotmail.com

${ }^{2}$ Ingeniero Agrónomo; Universidad de Córdoba; cmariosierra@hotmail.com
\end{abstract}


together with their parents were sown in the experimental area located at the University of Cordoba in Monteria - Colombia in 2009. Analysis of variance showed significant differences $(P<0,05)$ for the number of pods per plant, pods per meter, number of seeds per pod and grain yield, while for the seed weight differences were highly significant $(P<0,01)$. Otherwise, the precocity has not significance. The genetic variability present in the inbred lines allows select cultivars with good agronomic attributes and excellent yields of grain to improve production levels of farmers in the Sinú valley.

Key words: Cowpea, grain yield, landraces.

\section{INTRODUCCIÓN}

El fríjol caupí es una leguminosa de grano que ocupa el primer lugar en importancia económica en el Caribe colombiano, donde es cultivada por pequeños productores con limitaciones económicas y tecnológicas en superficies que oscilan entre 1000 y 10,000 $\mathrm{m}^{2}$; que se caracteriza por tener rusticidad, lo que le permite una buena adaptación a los diferentes sistemas de producción de las regiones semiáridas del trópico, es precoz y sus costos de producción son bajos (Araméndiz et al. 2003; Agronet 2010). Así mismo, es usada como abono verde dado su gran volumen de producción de biomasa y fijación de nitrógeno a través de sus nódulos radiculares y representa una importante fuente de proteína, calorías, ciertos minerales y vitaminas para los estratos sociales de reducida capacidad de compra de proteína animal (Obatolu 2003; Araméndiz et al. 2003; Xavier et al. 2005).

Se estima que 14 millones de hectáreas son cultivadas en todo el mundo, de las cuales 9,5 millones se realizan en el continente africano con rendimientos reducidos $\left(<600 \mathrm{~kg} \mathrm{ha}^{-1}\right)$, en razón al pobre potencial genético de los cultivares utilizados y por cambios en el régimen de Iluvias de las zonas productoras (Lopes et al. 2001). En Colombia para la cosecha del año 2008, fueron cultivadas en los departamentos de la Costa Atlántica alrededor de 14,457 ha con rendimiento de $600 \mathrm{~kg} \mathrm{ha}^{-1}$ (Agronet 2010).

Las poblaciones criollas de plantas autógamas se caracterizan por presentar variabilidad fenotípica como resultados de las mutaciones, recombinaciones genéticas y efectos ambientales sobre cada uno de los genotipos de la población, lo cual ha permitido la selección de genotipos adaptados a las condiciones climáticas específicas con características culinarias que son demandadas por los consumidores en el área de influencia. El fríjol caupí es originario de África y los centros de diversidad genética están ubicados en África e India (Phansak et al. 2005). Estudios de variabilidad genética usando marcadores moleculares RAPD adelantados por Xavier et al. (2005) demostraron la existencia de variabilidad genética cuando evaluaron 45 accesiones de fríjol caupí originarias de Brasil, Estados Unidos y Nigeria. Las procedentes de Brasil, presentaron una limitada base genética para alcanzar progresos significativos en el mejoramiento genético de esta especie. De igual manera, Phansak et al. (2005) encontraron diversidad genética entre 15 accesiones procedentes de Tailandia, Bangladesh, China, Laos, Filipinas y Taiwán, con el uso de marcadores microsatélites.

Esfuerzos para incrementar el rendimiento de 
grano de fríjol caupí ameritan el desarrollo de cultivares resistente a enfermedades, plagas y tolerancia a las nuevas condiciones climáticas resultantes del calentamiento global con precocidad suficiente que le permita adaptarse a las condiciones agroecológicas de los productores (Oyekanmi \& Sangodoyin 2007). El fríjol caupí es altamente susceptible a enfermedades virales y a una amplia gama de plagas como los trips, perforador de la vaina, áfidos, etc., que limitan el potencial de rendimiento que se estima entre 1,5 a 3,0 ha $^{-1}$ (Aliyu 2007).

Las características agronómicas de mayor importancia en el rendimiento de granos corresponden a número de ramas reproductivas por planta, número de vainas por planta, longitud de las vainas, número de semillas por vaina y peso de 100 semillas (Babalola 1980; Lopes et al. 2001, Deepa \& Balan 2006). Estudios sobre el comportamiento agronómico de líneas avanzadas de fríjol caupí adelantados por Tamgadge et al. (2008) en la India, destacan que el peso de las vainas, número de vainas por planta, diámetro de las vainas y peso de semillas por vainas poseen alta heredabilidad y en el mejoramiento simultáneo de esta especie, no debe de darse importancia a las características altura de planta y tamaño de las hojas.

A pesar de la importancia alimenticia de esta especie y adaptación a condiciones semiáridas, los programas de mejoramiento genético a nivel mundial son pocos, destacándose Brasil, Burkina Faso, India, Senegal y Estados Unidos, especialmente el IITA en Nigeria cuyo programa combina la obtención de cultivares de alto rendimiento de grano y resistencia a factores bióticos y abióticos (Singh et al.
2003). Lo antes anotado, torna esta especie interesante en el contexto regional y dada la reestructuración del sector agropecuario en 1992 por el gobierno nacional; este cultivo quedo al igual que otros de economía campesina, huérfano de investigación; lo cual justifica la búsqueda de nuevos cultivares con buenas características agronómicas. Por lo tanto, el objetivo de la presente investigación fue conocer el comportamiento agronómico de líneas seleccionadas de una población criolla de fríjol caupí, para su incorporación en pruebas regionales en la región Caribe y así contribuir al mejoramiento de los sistemas de producción.

\section{MATERIALES Y MÉTODOS}

Esta investigación se realizó durante el segundo semestre de siembra del año 2009 en el área experimental de la Universidad de Córdoba, Colombia la cual se encuentra localizada en Montería, departamento de Córdoba a $8^{\circ} 5^{\prime}$ de latitud Norte y $75^{\circ} 49^{\prime}$ de longitud Oeste y 20 m.s.n.m. En la zona predomina un régimen de precipitaciones con un promedio anual de $1,300 \mathrm{~mm}$, una temperatura promedio de $28^{\circ}$ C y 5,42 horas diarias de brillo solar (IGAC 1996).

Previa evaluación de 70 líneas obtenidas por selección individual en la población de fríjol caupí "Criolla", a través del diseño de Bloques Aumentados de Federer (1961), 12 líneas fueron seleccionadas por sus buenos atributos agronómicos y color de grano crema. Las líneas fueron enfrentadas a la población original (testigo) bajo un diseño experimental de bloques completos al azar (BCA), con cuatro repeticiones, para un total de 52 
unidades experimentales (UE). El tamaño de UE consistió de tres surcos de cuatro $\mathrm{m}$ de largo, con distancia entre plantas de $0,2 \mathrm{~m}$ y distancia entre surcos de 0,8 m. La siembra experimental se llevó a cabo mediante el sistema manual tecnificado colocando tres semillas al momento de la siembra y dejando una sola planta por sitio definitivo.

Las variables de respuesta consideradas en el estudio corresponden a: número de vainas por planta (VAPLAN), número de vainas por metro lineal (VAML), número de semillas por vaina (SEMVA), peso de semilla (PSEM), precocidad (PRECO) y rendimiento de grano (REND).

Con la información obtenida en campo se realizaron los respectivos análisis de varianza (ANDEVA) para cada variable con la ayuda del programa GENES versión Windows (2004.2.1), desarrollado por Cruz (2004), utilizando el siguiente modelo estadístico:

$$
Y_{i j}=\mu+T_{i}+B_{j}+\varepsilon_{i j}
$$

Donde: $Y_{\mathrm{ij}}=$ Efecto del i-ésimo tratamiento en la j-esima repetición; $\mu=$ media general, $T_{i}=$ efecto del i-ésimo tratamiento, $\mathrm{B}=$ efecto de la j-esima repetición, $\varepsilon_{\mathrm{ij}}=$ error aleatorio, con los supuestos usuales $\varepsilon_{\mathrm{ij}} \sim \mathrm{NI}\left(0, \sigma^{2}\right)$.
En aquellas variables en donde el análisis de varianza detectó diferencias significativas, se realizaron pruebas de separación de medias a través de la prueba de rango múltiple de Duncan.

\section{RESULTADOS Y DISCUSIÓN}

El análisis de varianza (Tabla 1) destaca que en términos generales diferencias significativas y altamente significativas en las líneas evaluadas, a excepción del carácter precocidad, resaltando con ello que las líneas estudiadas difieren en su composición genética. Así mismo, las presunciones del modelo matemático (independencia, homogeneidad de varianza, aditividad y normalidad) fueron satisfechas, indicando que las conclusiones derivadas del estudio son confiables.

La calidad de la precisión del experimento medida a través del coeficiente de variación experimental de según Estefanel et al. (1987); Silva et al. (2002); Ribeiro et al. (2004), se considera como media para las variables rendimiento de grano, precocidad, número de vainas por planta y número de vainas por metro lineal por encontrarse en la faja de 14\% y $26,5 \%$ y alta para número de semillas por vaina y peso de cien semillas, por ser inferior

Tabla 1. Cuadrados medios de seis características agronómicas en líneas avanzadas de fríjol caupí en el Valle del Sinú.

\begin{tabular}{lccccccc}
\hline \multirow{2}{*}{$\begin{array}{c}\text { Fuente de } \\
\text { variación }\end{array}$} & \multirow{2}{*}{ G.L. } & \multicolumn{7}{c}{ Cuadrados medios } \\
\cline { 3 - 8 } & & VAPLAN $^{1}$ & VAML $^{2}$ & SEMVA $^{3}$ & PSEM $^{4}$ & PRECO $^{\mathbf{5}}$ & REND $^{\mathbf{6}}$ \\
\hline Bloques & 3 & 34,18 & 176,15 & 0,35 & 3,26 & 184,18 & 2664,9 \\
Tratamientos & 12 & $27,17^{*}$ & $628,10^{*}$ & $2,46^{*}$ & $22,56^{* *}$ & $69,22^{\text {ns }}$ & $49941,1^{*}$ \\
Error & 36 & 12,94 & 284,87 & 1,43 & 2,20 & 111,38 & 23423,1 \\
\hline
\end{tabular}

${ }^{1}$ VAPLAN: número de vainas por planta; ${ }^{2}$ VAML: vainas por metro lineal; ${ }^{3}$ SEMVA: número de semillas por vaina; ${ }^{4}$ PSEM: peso de cien semillas; ${ }^{5}$ PRECO: número de días a cosecha; ${ }^{6}$ REND: kilogramos por hectárea; ${ }^{* *}$ : significativo al $1 \%$ de probabilidad; *: significativo al 5\% de probabilidad y ns: no significativo. 
a 14\%. Así mismo, los valores reportados en el presente estudio, resultaron inferiores a los obtenidos por Hegde \& Mishra (2009) en estudios similares.

\section{Número de vainas por planta}

El mayor número de vainas por planta se presentó en las líneas L066, L002 y L019 con 21,$1 ; 20,0$ y 19,6, respectivamente (Tabla 2), las cuales superaron al testigo que registró 12 vainas, en un $75,8 \%, 66,6 \%$ y $63,3 \%$, lo que constituye una ganancia significativa $y$ confirmada en el análisis de variancia (Tabla 1). Considerando la correlación genética positiva que existe entre esta variable y el rendimiento de grano reportada por Lopes et al. (2001); Deepa \& Balan (2006) y Srivastava et al. (2008), confirman que el número de vainas por planta es uno de los componentes del rendimiento más importante y con efecto directo significativo en el rendimiento de grano, por lo cual se espera una mayor eficiencia en la selección de las líneas de mejor respuesta.

Los resultados anteriores corroboran lo manifestado por Hawtin et al. (1996) y Hegde \& Mishra (2009), quienes señalan que las poblaciones criollas de fríjol caupí, poseen características importantes y de gran utilidad, para el desarrollo de cultivares adaptados localmente de alto rendimiento y resistencia a factores bióticos y abióticos.

\section{Número de vainas por metro lineal}

En atención a los valores de la prueba de media (Tabla 2), la población original registró el menor número de vainas con 47; superada por las líneas L033, L003, L031, L002 y L019, que mostraron guarismos de 86,0; 84,5; 84,3; 82,3 y 81,0 vainas, respectivamente, lo que

Tabla 2. Valores medios de siete características de interés agronómico en 13 genotipos de fríjol caupí en el Valle del Sinú.

\begin{tabular}{lllllll}
\hline Tratamiento & $\begin{array}{c}\text { VAPLAN } \\
(\#)\end{array}$ & $\begin{array}{c}\text { VAML }^{2} \\
(\#)\end{array}$ & $\begin{array}{c}\text { SEMVA }^{3} \\
(\#)\end{array}$ & $\begin{array}{c}\text { PSEM }^{4} \\
(\mathbf{g})\end{array}$ & $\begin{array}{c}\text { REND }^{\mathbf{5}} \\
\left.\mathbf{( k g ~ h a}^{-1}\right)\end{array}$ & $\begin{array}{c}\text { PRECO }^{\mathbf{6}} \\
\text { (días) }^{\mathbf{2}}\end{array}$ \\
\hline L066 & $21,4 \mathrm{a}$ & $74,3 \mathrm{ab}$ & $10,7 \mathrm{ab}$ & $19,8 \mathrm{ab}$ & $417,9 \mathrm{c}$ & $68,4 \mathrm{a}$ \\
L002 & $20,1 \mathrm{a}$ & $82,3 \mathrm{a}$ & $9,0 \mathrm{~b}$ & $21,4 \mathrm{a}$ & $604,1 \mathrm{abc}$ & $63,4 \mathrm{a}$ \\
L019 & $19,6 \mathrm{a}$ & $81,0 \mathrm{a}$ & $11,9 \mathrm{a}$ & $15,0 \mathrm{c}$ & $789,5 \mathrm{a}$ & $63,3 \mathrm{a}$ \\
L031 & $19,3 \mathrm{ab}$ & $84,3 \mathrm{a}$ & $11,0 \mathrm{a}$ & $20,3 \mathrm{ab}$ & $767,7 \mathrm{a}$ & $68,7 \mathrm{a}$ \\
L070 & $18,3 \mathrm{ab}$ & $70,8 \mathrm{abc}$ & $10,9 \mathrm{ab}$ & $20,2 \mathrm{ab}$ & $445,5 \mathrm{bc}$ & $75,3 \mathrm{a}$ \\
L033 & $17,8 \mathrm{abc}$ & $86,0 \mathrm{a}$ & $10,7 \mathrm{ab}$ & $19,8 \mathrm{ab}$ & $535,3 \mathrm{abc}$ & $71,9 \mathrm{a}$ \\
L003 & $17,7 \mathrm{abc}$ & $84,5 \mathrm{a}$ & $11,0 \mathrm{ab}$ & $19,5 \mathrm{ab}$ & $695,2 \mathrm{ab}$ & $72,6 \mathrm{a}$ \\
L056 & $17,4 \mathrm{abc}$ & $78,5 \mathrm{a}$ & $11,8 \mathrm{a}$ & $14,2 \mathrm{c}$ & $643,5 \mathrm{abc}$ & $65,5 \mathrm{a}$ \\
L047 & $17,3 \mathrm{abc}$ & $70,5 \mathrm{abc}$ & $10,7 \mathrm{ab}$ & $18,9 \mathrm{~b}$ & $644,5 \mathrm{abc}$ & $65,7 \mathrm{a}$ \\
L026 & $16,1 \mathrm{abc}$ & $78,5 \mathrm{a}$ & $10,9 \mathrm{ab}$ & $14,9 \mathrm{c}$ & $566,6 \mathrm{abc}$ & $63,1 \mathrm{a}$ \\
L037 & $16,1 \mathrm{abc}$ & $78,0 \mathrm{a}$ & $10,1 \mathrm{ab}$ & $16,5 \mathrm{c}$ & $644,0 \mathrm{abc}$ & $64,4 \mathrm{a}$ \\
L055 & $13,3 \mathrm{bc}$ & $49,5 \mathrm{bc}$ & $11,9 \mathrm{a}$ & $19,8 \mathrm{ab}$ & $506,8 \mathrm{bc}$ & $62,7 \mathrm{a}$ \\
Testigo & $12,1 \mathrm{c}$ & $47,0 \mathrm{c}$ & $10,6 \mathrm{ab}$ & $18,6 \mathrm{~b}$ & $618,7 \mathrm{abc}$ & $64,2 \mathrm{a}$ \\
$\tilde{\chi}$ & 17,3 & 74,2 & 10,8 & 18,4 & 606,0 & 66,9 \\
C.V. $(\%)$ & 20,6 & 22,7 & 11 & 8,08 & 15,78 & 25,2 \\
\hline
\end{tabular}

${ }^{1}$ VAPLAN: número de vainas por planta; ${ }^{2}$ VAML: vainas por metro lineal; ${ }^{3}$ SEMVA: número de semillas por vaina; ${ }^{4}$ PSEM: peso de cien semillas; ${ }^{5}$ REND: kilogramos por hectárea; ${ }^{6}$ PRECO: número de días a cosecha. 
equivale a una ganancia de $82,9 \% ; 79,7 \%$; $79,3 \% ; 75,1 \%$ y $72,3 \%$ y corrobora con ello, las diferencias genéticas entre las diferentes líneas sometidas a estudio.

Estas diferencias pueden obedecer al efecto de las altas temperaturas en la emisión y viabilidad de polen, lo que conduce al aborto de flores y poca formación de vainas y peso de granos, como lo sostienen Rajendiran y Ramanujam (2004) y Singh et al. (2010).

\section{Número de semillas por vaina}

Las líneas L019, L055, L056, L003 y L031 (Tabla 2) mostraron el mayor número de semillas por vaina con 11,$9 ; 11,9 ; 11,8 ; 11,0$ y 11,0 , semillas respectivamente. La línea L002 pese a mostrar altos valores con relación al número de vainas por planta y número de vainas por metro lineal, mostró el menor número medio de semillas (9), lo que puede obedecer a la competencia en la distribución de los fotosintatos en torno a la capacidad de la planta de satisfacer los diferentes vertederos. Sin embargo, ésta mostró el mayor peso de semillas (21,4 g); lo que es un factor positivo de la línea en el programa de mejoramiento genético. Esta respuesta diferencial entre los genotipos estudiados, coincide con lo reportado con Singh et al. (2010) quienes sostienen la existencia de un alto grado de variación genética en caracteres vegetativos y reproductivos en poblaciones criollas, lo que permite la selección de genotipos para condiciones agroecológicas específicas de acuerdo a Hawtin et al. (1996) y Hegde \& Mishra (2009).

\section{Peso de semillas}

El peso de semilla de las líneas L002, L031 y L070, resultó superior a la población original en un $15,0 \% ; 9,1 \%$ y 9,0\%, respectivamente, lo que representa una ganancia significativa por su correlación positiva con el rendimiento de grano de acuerdo a Deepa \& Balan (2006) y bastante confiable, por su alta heredabilidad en sentido amplio y estrecho reportada por Padi (2007).

Dado que el precio en el mercado internacional de este grano, está en función del tamaño, color y textura del grano, color del hilium y daño de los mismo; estudios económicos realizados por Mishili et al. (2009), indican que el aumento en el tamaño de grano, reflejó una mejora de los precios al pasar de \$ US 0,0261 a \$ US 0,0047 por kilogramo extra, con pesos de semillas de 18,5g. La existencia de líneas con peso de semilla superior a los 20 g, constituye un progreso en la calidad de grano para los productores y consumidores de esta especie, ya que ello conduce a un mayor almacenamiento de proteína.

\section{Precocidad}

La precocidad no registró diferencias en los días a cosecha entre las líneas en evaluación y la población original. El hecho de tener genotipos de semejante comportamiento al material original, favorece los requisitos de los agricultores; dado que la agricultura está siendo afectada por el cambio climático, degradación ambiental, crecimiento poblacional y déficit hídrico, especialmente en el trópico donde son muy erráticas y se demandan nuevas variedades con adaptación a estos nuevos ambientes como lo resaltan Agbicodo et al. (2009). 


\section{Rendimiento de granos}

Las líneas con mayor rendimiento de grano con respecto a su población parental fueron L019, L031 y L003 con 789,5; 695; 2 y $767,7 \mathrm{~kg} \mathrm{ha-1}$ respectivamente, superando significativamente $(P \leq 0,05)$ el rendimiento medio del tratamiento testigo $\left(618,7 \mathrm{~kg} \mathrm{ha}^{-1}\right)$. Otras líneas como la L037, L047 y L056 se destacan también por superar al testigo, más no difieren estadísticamente (Tabla 1).

La razón de un mayor rendimiento de grano de las líneas se sustenta en los componentes del rendimiento: número de vainas por planta, vainas por metro lineal y peso de semillas, que poseen alta correlación genética con el rendimiento (Deepa \& Balan 2006; Srivastava et al. (2008); Samad \& Lavanya 2005) y posiblemente al uso eficiente de la radiación en la fase reproductiva, ya que valores de correlación positivos de $0,85^{* *}$ a $0,89 * *$ reportados por Tesfaye et al. (2006) así lo destacan con respecto al rendimiento de grano; dado que altos índices de área foliar permiten reducir la pérdida de agua e interceptan y convierten la radiación en materia seca mucho más eficiente hacia la producción de granos Tesfaye et al. (2006).

\section{CONCLUSIONES}

Las diferencias en el comportamiento agronómico de las líneas con respecto a la población parental, resaltan la existencia de variabilidad genética en la población original. Las líneas seleccionadas promisorias L019, L031 y L003, por su buen comportamiento agronómico y rendimiento de grano, pueden ser sometidas a valoración en diferentes ambientes para conocer su estabilidad fenotípica.
Es posible direccionar la selección hacia la obtención de materiales con mayores número de semillas por vaina y peso de semillas para mejorar el rendimiento de nuevos cultivares con adaptación a las condiciones del Valle del Sinú.

\section{REFERENCIAS}

\section{Agbicodo, E.M., Fatokun, C.A., Muranaka, S.,} Visser, R.F.F. and Linden van der, C. G. 2009. Breeding drought tolerant cowpea: constraints, accomplishments, and future prospects. Euphytica 167 (3):353-370.

Agronet 2010. Estadística de sistemas de producción agrícola. http.//www. agronet.gov.co [20 Febrero 2010].

Aliyu, B. 2007. Heritability and gene effects for incorporating pubescence into cowpea (Vigna unguiculata (L) Walp.) from V.rhomboidea Burtt. Davy. Euphytica 155(3):295-303.

Araméndiz, H., Robles, J. y Cabrales, R. 2003. Caracterización del fríjol caupí (Vigna unguiculata (L.) Walp) por su contenido de proteína. Fitotecnia Colombiana 3(2):17-23.

Babalola, O. 1980. Growth and development of three varieties of cowpea in western Nigeria. Yiel and dry matter production. Tropical Grain Legume Bull 20(1):3-5.

Cruz, C.D. 2004. Programa Genes: versão Windows (2004.2.1): aplicativo computacional em genética e estatística. UFV, Viçosa, Brasil. 
Deepa, S.N. and Balan, A. 2006. Correlation studies in cowpea (Vigna unguiculata (L.) Walp). Madras Agric. J.93 (7-12): 260262. p648.

Estefanel, V., Pignataro, I.A.B e Storck, L. 1987. Avaliação do coeficiente de variação de experimentos com algumas culturas agrícolas. In: SIMPÓSIO DE ESTATÍSTICA APLICADA À EXPERIMENTAÇÃO AGRONÔMICA, Londrina. Anais Londrina: DME/CCE/Universidade Estadual de Londrina, p115-131.

Federer, W. T. 1961. Augmented designs with one-way elimination of heterogeneity. Biometrics 17(3): 447-473.

Hawtin, G., Iwanaga, M. and Hodgkin, T. 1996. Genetic resources in breeding for adaptation. Euphytica 92(2):255-266.

Hegde, V.S. and Mishra, S.K. 2009. Landraces of cowpea, Vigna unguiculata (L.) Walp., as potential sources of genes for unique characters in breeding. Genet Resour Crop Evol 56:615-627.

\section{INSTITUTO GEOGRÁFICO AGUSTÍN} CODAZZI (IGAC), 1996. Diccionario geográfico de Colombia. Tomo 3. Tercera edición, Bogotá, p1858.

Lopes, A.C.A., Freire Filho, F.R., Silva, R.B., Campo, F.C. e Rocha, M.M. 2001. Variabilidade e correlacões entre caracteres agronômicos em caupí (Vigna unguiculata (L.) Walp). Pesq. agropec. bras 36(3):515-520.
Mishili, F.J., Fulton, J., Shehu, M., Kushwaha, S., Marfo, K., Jamal, M., Kergna, A. and Lowenberg-DeBoer, J. 2009. Consumer Preferences for Quality Characteristics Along the Cowpea Value Chain in Nigeria, Ghana, and Mali. Agribusiness 25(1):16-35.

Obatolu, V.A. 2003. Growth pattern of infants fed with a mixture of extruded malted maize and cowpea. Nutrition 19(2):174178.

Oyekanmi, A.A. and Sangodoyin, O.S. 2007. Evaluation of advances lines of cowpea (Vigna unguiculata (L.) Walp) for agronomic traits and grain yield in the transition zone of Nigeria. Asian Journal of Plant Sciences 6(1):163-167.

Padi, F.K. 2007. Early generation selection for high yielding cowpea genotypes in additive series intercropping systems with sorghum. Annals of Applied Biology 151(3): 391-400.

Phansak, P.P., Taylor, P.W.J. and Mongkolporn, O. 2005. Genetic diversity in yardlong bean (Vigna unguiculata ssp. sesquipedalis) and related Vigna species using sequence tagged microsatellite site analysis. Scientia Horticulturae 106 (1):137-146.

Rajendiran, K. and Ramanujam, M.P. 2004. Improvement of biomass partitioning, flowering and yield by triadimefon in UV-B stressed Vigna radiata (L.) Wilczek, Biol. Plant 48(1): 145-148. 
Ribeiro, N.D., Cargnelutti, A., Hoffmann, L.e Borba, S. 2004. Precisão experimental na avaliação de cultivares de feijão de diferentes hábitos de crescimento. Ciência Rural 34 (5):1371-1377.

Samad, S.S. and Lavanya, G.R. 2005. Selection Strategy for yield improvement in Mungbean (Vigna radiata (L.) Wilczek. Madras Agric. J 92(7-9): 526-528.

Silva, F.B., Bruzi, A.T. e Ramalho, M.A.P. 2002. Precisão experimental na avaliação de cultivares de feijão. In: CONGRESSO NACIONAL DE PESQUISA DE FEIJÃO, 7., Viçosa, 2002. Anais Viçosa: UFV, p288-291.

Singh, B.B., Ajeigbe, H.A., Tarawali, S.A., Fernández-Rivera, S. and Abubakar, $M$. 2003. Improving the production and utilization of cowpea as food and fodder. Field Crops Research 84(1-2):169-177.

Singh, S.K., Kakani, V.G., Surabhi, G.K. and Reddy. K.R. 2010. Cowpea (Vigna unguiculata [L.] Walp.) genotypes response to multiple abiotic stresses. Journal of Photochemistry and Photobiology B: Biology 100(2):135146.
Srivastava, A., Lavanya, G.R., Pandey, R.K. and Rastogi, M.C. 2008. Association and cause-and-effect analysis in F2 generation of mungbean (Vigna radiata (L.) Wilczek). Madras Agric. J.95 (1-6): 195-199.

Xavier, G.R., Martins, L.M., Rumjanek, N.G. e Freire Filho, F.R. 2005. Variabilidade genética em acessos de caupí analisada por meio de marcadores RAPD. Pesq. agropec. bras 40 (4):353-359.

Tamgadge, S., Dod, V.N., Mahorkar, V.K. and Peshattiuar, P.D. 2008. Genetic variability, heritability and genetic advance in cowpea [Vigna unguiculata (L.) Walp]. Asian Journal of Agricultural 3(1):30-32.

Tesfaye, K.; Walker, S. and Tsubo, M. 2006. Radiation interception and radiation use efficiency of three grain legumes under water deficit conditions in a semiarid environment. European Journal of Agronomy 25(1):60-70. 